\title{
Strong and Tough PAm/SA Hydrogel with Highly Strain Sensitivity
}

\author{
Lingqin Zhang, Qifeng Jiang, Yiming Zhao, Jie Yuan, Xiaoying Zha, Haojiang Xie, Fankai Kong and \\ Xingliang Xiong*
}

Department of Medical Information College, Chongqing Medical University, Chongqing, 400016, China

${ }^{*}$ Corresponding Author: Xingliang Xiong. Email: xlxiong@cqmu.edu.cn

Received: 15 March 2021 Accepted: 10 May 2021

\begin{abstract}
The hydrogel is a preferred material for flexible wearable sensors. In practical application, it should have highefficiency mechanical toughness and self-healing performance. Besides, hydrogel requires good affinity and adhesion because of its contact with the skin. In this experiment, we made an ultra-tough hydrogel with excellent cell affinity and adhesion. We used sodium alginate (SA) and polyacrylamide (PAm) mixture as a flexible base fluid. Polydopamine reduce graphene oxide (prGO) was used as conductive nanofiller, and then PAm-prGO-SA semiinterpenetrating network hydrogel was formed through Am radical polymerization. The presence of prGO endows the hydrogels with excellent electrical conductivity. Simultaneously, some non-reduced GO forms non-covalent cross-links with PAm, SA, and Polydopamine (PDA) in the hydrogel network. The stress of PAm-prGO-SA hydrogel can reach $750 \mathrm{KPa}$, and the strain is $900 \%$. The hydrogel, combined with its excellent electrical, mechanical properties, and biocompatibility, is expected to be applied in portable, remote, and real-time health monitoring systems.
\end{abstract}

\section{KEYWORDS}

Sodium alginate; polyacrylamide; graphene oxide; nanofiller

\section{Introduction}

Conductive hydrogel is a conductive polymer material rich in water and has a three-dimensional network microstructure [1,2]. It has attracted much attention due to its great potential for use in actuators [3], flexible electronics [4], and Biomedicine [5,6]. At present, the conductive hydrogels that have been developed still have problems. Firstly, the poor strength and low tensile ratio make the flexible conductive sensor unable to endure strenuous activity. Secondly, the adhesion of flexible conductive sensors is lacking [7-9]. Conductive hydrogels need to be fixed at the monitoring sites with bandages and other materials. It not only reduces the wearability of conductive hydrogels, but also reduces the comfort of flexible conductive sensors. Moreover, excellent mechanical properties and high adhesion are difficult to coexist [10]. Therefore, mechanical properties and high adhesion provide difficulty in their coexistence [11]. It is an essential issue in hydrogel development. Thirdly, the poor moisturizing properties make hydrogels more rigid, less adhesion, and shorter service life [12]. Finally, the poor dispersion of fullerenebased conductive materials limits its application [13]. Therefore, many conductive hydrogels have ionic conductance, which reduces the conductivity and sensitivity of conductive hydrogels. These disadvantages restrict the comfort and practicability of the conductive hydrogel. 
As a common material, Polyacrylamide (PAm) has attracted much attention due to its excellent mechanical properties $[14,15]$. However, PAm has poor water retention and adhesion, which limits its application. Sodium Alginate (SA) with high viscosity and moisture retention can compensate for the defects of PAm as a purely natural material. In the literature, PAm and SA are mixed to form hydrogels through the "one-pot" method [16]. The combination of PAm and SA not only enhances the mechanical properties of hydrogels, but also endows water retention and adhesion. Therefore, hydrogels have good tissue adhesion ability, which can solve the low accuracy of sensors caused by insufficient contact.

As a member of the Graphene family, Graphene Oxide (GO) has good dispersion [17]. However, due to the poor electrical properties of GO, it must be reduced to reduced Graphene Oxide (rGO) to improve its electrical properties. Yang et al. [18] reported that dopamine (DA) can self-polymerize in an alkaline environment to form polydopamine (PDA) and reduce GO to polydopamine reduce Graphene Oxide (prGO). The prGO obtained by PDA reduction can not only be wholly dispersed in hydrogels, but also has excellent conductivity and sensitivity due to the reduction rate of $51 \%$. Meanwhile, the Mussel-like properties of polydopamine also enhance the adhesion and water retention of hydrogels [19].

In this experiment, PAm and SA were used as flexible substrate materials and $\mathrm{rGO}$ as conductive materials. Polydopamine (PDA) was used as a reducing agent and binder to make a conductive hydrogel with a semi-interpenetrating network structure. Through many application tests, it can be determined that the conductive hydrogel has excellent electrical properties, mechanical properties, biocompatibility, adhesion, and moisture retention ability under the action of hydrogen bonding and Van Der Waals Force. This hydrogel is expected to be applied in portable, remote, and real-time health monitoring systems.

\section{Experiment Section}

\subsection{Materials}

GO was purchased from Nanjing Jicang Nanotechnology Co., Ltd., Nanjing, China. Acrylamide (Am), Ammonium persulfate (APS), Sodium alginate (SA), N, N'-methylenebis-acrylamide (BIS) and N, N, N', $\mathrm{N}^{\prime}$-tetramethylethylenediamine (TEMED) were obtained from Aladdin. DA and sodium borate decahydrate were provided by BBI Co., Ltd., Shanghai, China. Cell Counting kit-8 (CCK-8) and Fetal Bovine Serum was obtained from Solarbio Life Science Co., Ltd., Beijing. Trypsin was purchased from Thermo Fisher Scientific.

\subsection{Preparation of Hydrogels}

(1) Partial reduction of GO by PDA: disperse GO in ultra-pure water, and form uniform GO suspension after ultrasonic dispersion for $40 \mathrm{~min}$. Then add dopamine hydrochloride into the suspension, adjust $\mathrm{pH}$ to 11 with $0.1 \mathrm{M} \mathrm{NaOH}$ solution; keep stirring for $2 \mathrm{~h}$ at $65^{\circ} \mathrm{C}$. The $\mathrm{GO}$ in the solution is partially reduced to prGO to obtain a mixed liquid of prGO nano conductive filler.

(2) Preparation of PAm-prGO-SA mixed solution: SA is pre-soaked in ultrapure water for $8 \mathrm{~h}$. Then, the vigorous stirring was continued for $2 \mathrm{~h}$ under a temperature of $70^{\circ} \mathrm{C}$, and the stirring speed was 300 $\mathrm{rpm}$. Add Am monomer to the SA solution and mix to obtain a flexible substrate solution. Add the prGO nano conductive filler mixture prepared in Step (1) to the flexible substrate solution. After sufficient vigorous stirring, it was left in a vacuum oven for $4 \mathrm{~h}$ to remove bubbles.

(3) Formation of PAm-prGO-SA hydrogel: remove the mixed liquid after defoaming, add APS, MBA, TMEDA, and quickly stir under ice bath conditions to make it evenly cross-linked to form graphenebased conductive hydrogel glue. Finally, the cross-linked uniform hydrogel is connected to the analyzer through copper tape to assemble a flexible sensor.

The ratio of ingredients used in the preparation of PAm-prGO-SA hydrogel in this article (Tab. 1). 
Table 1: The compositions of PAm-prGO-SA hydrogel

\begin{tabular}{llllllll}
\hline Hydrogel & $\begin{array}{l}\text { Am } \\
\text { (wt.\%) }\end{array}$ & $\begin{array}{l}\text { SA } \\
\text { (wt.\%) }\end{array}$ & $\begin{array}{l}\text { GO } \\
\text { wt. } \%\end{array}$ & $\begin{array}{l}\text { GO/ } \\
\text { DA }\end{array}$ & $\begin{array}{l}\text { MBA } / \\
\text { Am wt.\% }\end{array}$ & $\begin{array}{l}\text { APS/ } \\
\text { Am wt.\% }\end{array}$ & $\begin{array}{l}\text { TMEDA/ } \\
\text { Am wt. } \%\end{array}$ \\
\hline PAm-SA & 20 & 0 & 0 & 0 & 0.2 & 1 & 0.2 \\
& 20 & 1 & 0 & 0 & 0.2 & 1 & 0.2 \\
& 20 & 2 & 0 & 0 & 0.2 & 1 & 0.2 \\
PAm-prGO-SA & 20 & 2 & 0 & 0 & 0.2 & 1 & 0.2 \\
& 20 & 2 & 0.15 & 1.5 & 0.2 & 2 & 0.4 \\
& 20 & 2 & 0.2 & 1.5 & 0.2 & 3 & 0.6 \\
& 20 & 2 & 0.25 & 1.5 & 0.2 & 3 & 0.6 \\
PAm-GO-SA & 20 & 2 & 0.2 & 0 & 0.2 & 1 & 0.2 \\
& 20 & 2 & 0 & 0.01 & 0.2 & 3 & 0.4 \\
\hline
\end{tabular}

\subsection{Characterization}

The morphology of hydrogels was characterized by a scanning electron microscope (Hitachi s-3000N FE-SEM). Before SEM characterization, the hydrogel was frozen at $-80^{\circ} \mathrm{C}$ for $24 \mathrm{~h}$ and then transferred to a vacuum dryer for drying. FTIR spectra were obtained from a Nicolet iS50 NIR FTIR instrument and microscopic images were obtained from Leica DMi8 inverted fluorescence microscope.

\subsection{Swelling Ratio Test}

The prepared hydrogel (PAm, PAm-SA, PAm-GO-SA, PAm-PDA-SA, PAm-prGO-SA) was dried in a vacuum drying box to constant weight $\left(\mathrm{W}_{0}\right)$. After swelling at room temperature in $\mathrm{PBS}$ solution $(\mathrm{PH}=7.4)$ until constant weight, the excess PBS of the remaining surface was wiped by tissue paper and the mass of the swelling equilibrium was measured (Wa). The percentage of equilibrium swelling ratio (SR) of PAm-prGOSA hydrogel was obtained by the equation $\left(\mathrm{SR}=\left(\mathrm{W}_{\mathrm{a}}-\mathrm{W}_{0}\right) / \mathrm{W}_{0} \times 100 \%\right)$. Among them, $\mathrm{W}_{0}$ is the quality of hydrogel before swelling.

\subsection{Water Retention}

The liquid retention was probed by putting the swelled hydrogel (PAm-SA, PAm-GO-SA, PAm-PDASA, PAm-prGO-SA) was placed in an incubator with a relative humidity of $50 \%$ at 25 degrees. At the beginning and at each time step, The hydrogel weighing $\left(\mathrm{W}_{\mathrm{b}}\right)$ was measured, and the water retention $\left(\mathrm{W}_{\mathrm{R}}\right)$ of PAm-prGO-SA hydrogel was calculated by the equation $\left(\mathrm{W}_{\mathrm{R}}=\left(\mathrm{W}_{\mathrm{b}}-\mathrm{W}_{0}\right) / \mathrm{W}_{0} \times 100 \%\right)$. Among them, $\mathrm{W}_{0}$ is the quality of hydrogel before drying until it is constant.

\subsection{Mechanical Test}

Tensile, Cyclic, and adhesion tests were carried out on a universal testing machine (E43.104, MTS, US), the displacement rate was 50,10 and $2 \mathrm{~mm} \mathrm{~min}^{-1}$, respectively, the size of the samples in all the tests was 20 $\mathrm{mm} \times 10 \mathrm{~mm} \times 5 \mathrm{~mm}$. 


\subsection{Conductive Performance Tests}

The conductivity of hydrogels of different GO content was determined with an electrochemical workstation (CHI604E, CH Instruments Inc., China) by the two-probe method. A strain sensor based on hydrogel was connected to the electrochemical workstation through a copper strip. To monitor human motion, the assembled sensors were attached to the wrist, neck, knee, and chest, and recorded the output impedance curves of cyclic motion under different amplitudes.

\subsection{Cytotoxicity Evaluation}

The CCK-8 method was used to analyze the cytotoxicity of the PAm-prGO-SA hydrogel to NIH3T3 fibroblasts. The supernatant of PAm-prGO-SA hydrogel was used as the medium of the experimental group. Place the PAm-prGO-SA hydrogel in the culture medium and soak for $48 \mathrm{~h}$, then centrifuge and take the supernatant for later use. The control group used the DEME medium, and each group had 9 pore samples. The samples were placed in the incubator and incubated for $2 \mathrm{~h}$. Then the optical density (OD) value at $450 \mathrm{~nm}$ was measured by enzyme-linked immunosorbent assay. The relative growth rate of cells was calculated by reading OD values to determine the proliferation of cells and to investigating the biocompatibility of PAm-prGO-SA hydrogels.

\section{Results and Discussion}

The facile combined "One-Pot" preparation process of high toughness and electro-conductive PAm-prGO-SA hydrogel hydrogels with a uniform 3D-network structure is schematically presented in Fig. 1. It mainly consists of three steps. Firstly, GO was added in the aqueous system with $\mathrm{pH}=$ 10-11 following an ultrasonication treatment, then DA was added into GO suspension to make DA self-polymerize to synthesize PDA. PDA can reduce GO to prGO in an alkaline environment. Subsequently, SA and Am were added into the mixed suspension and stirred for $1 \mathrm{~h}$ before vacuum defoaming. Thirdly, APS, MBA, and TMEDA were added to the solution in turn under rapid stirring, and then the gel was formed by standing. PAm-prGO-SA hydrogels is a semi IPN hydrogel structure. The PAm chain forms a covalently crosslinked network after MBA crosslinking, giving the PAm-prGO-SA hydrogel excellent toughness. SA chain contains a large number of hydrophilic groups, it can help hydrogel lock the internal moisture. Therefore, the hydrogel has excellent water retention properties. Because of the presence of the prGO, the hydrogel has excellent electrical properties. Strong interactions between GO and PAm, PDA and SA chains, such as hydrogen bonds, $\pi-\pi$ bonds, and intermolecular interactions, further enhance the toughness of PAm-prGO-SA hydrogels [20].

\subsection{Microstructures and Chemical Analysis}

As shown in the scanning electron microscope (SEM) image of Fig. 2, the composite hydrogels of different components all present a typical porous structure. The formation of a well-organized network without obvious agglomeration indicated that the composite hydrogels have good swelling properties. After PDA is introduced into the hydrogel network, because PDA has adhesiveness, cyst-like attachments can be seen on the surface of PAm-PDA-SA. Thanks to the ideal architecture of PAm-prGO-SA hydrogel, the internal stress could be effectively transferred from the 3D network under external force and provided supplemental energy dissipation ability, thus enhancing the mechanical strength of the composite hydrogels [21]. 


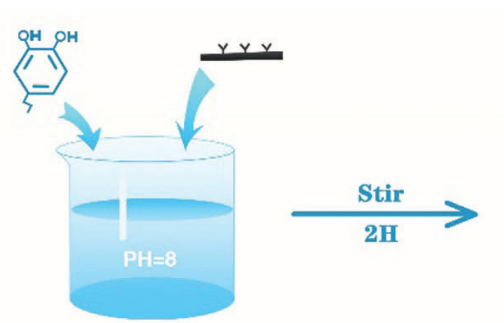

(A)

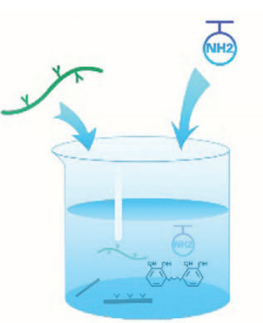

(B)

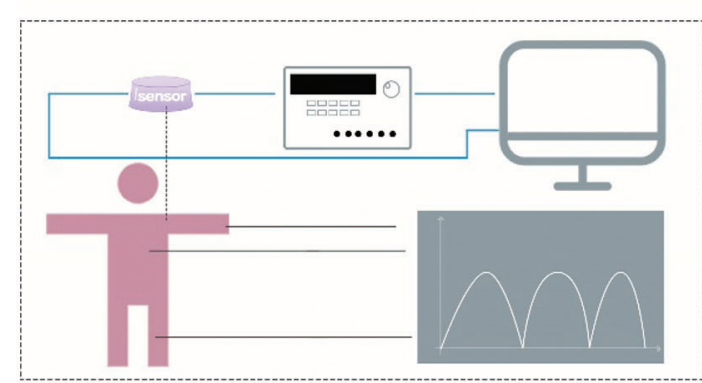

(C)

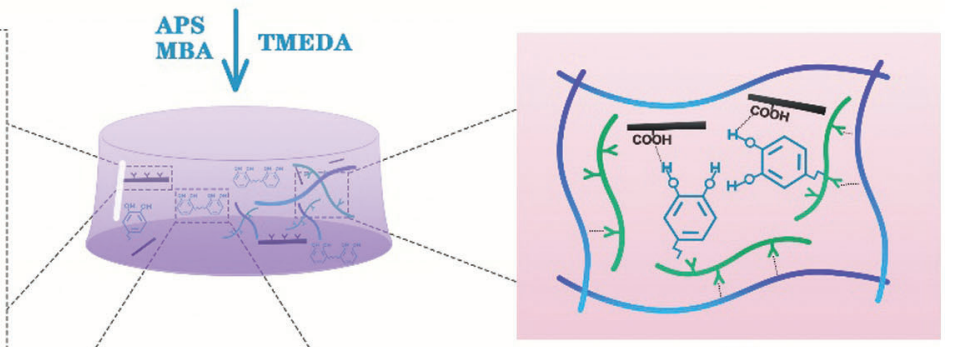

(E)

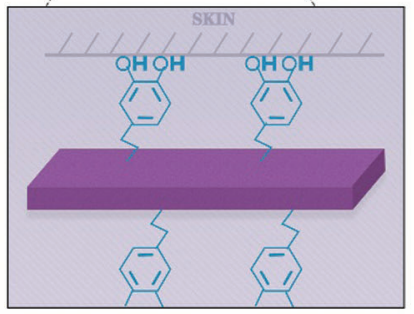

(D)
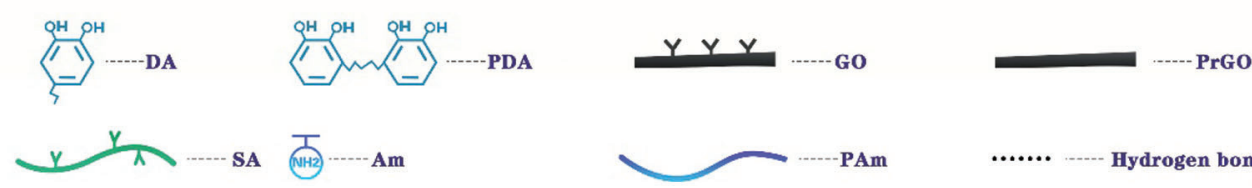

Figure 1: Schematic diagram of preparation of PAm-prGO-SA hydrogel. (A) Self-polymerization of dopamine. (B) The "one-pot method" prepares PAm-prGO-SA hydrogel. (C) The application of PAmprGO-SA hydrogel. (D) The adhesion principle of PAm-prGO-SA hydrogel. (E) The internal structure of PAm-prGO-SA hydrogel

Fig. 3a shows the infrared spectra of GO, prGO, PAm, and SA in the wavelength range of $400-4000 \mathrm{~cm}^{-1}$. The tensile vibration peak of the $\mathrm{OH}$ group of $\mathrm{GO}$ and $\mathrm{SA}$ appeared at around $3400 \mathrm{~cm}^{-1}$, while the prGO emerged at $3218 \mathrm{~cm}^{-1}$ [22-24]. In the GO spectrum, the characteristic peak of $\mathrm{C}=\mathrm{O}$ stretching vibration in $-\mathrm{COOH}$ appears at about $1627 \mathrm{~cm}^{-1} .1216 .97 \mathrm{~cm}^{-1}$ is due to $\delta \mathrm{H}-\mathrm{O}-\mathrm{H}$ tensile vibration [25]. In the SA spectrum, the saturated $\mathrm{C}-\mathrm{H}$ stretching vibration appears in the band around $2929 \mathrm{~cm}^{-1}$. About $1612 \mathrm{~cm}^{-1}$ and $1421 \mathrm{~cm}^{-1}$ responsible for the asymmetric stretching vibration and symmetric stretching vibration. The peak at $1033 \mathrm{~cm}^{-1}$ confirming the presence of the $\mathrm{C}-\mathrm{O}-\mathrm{C}$ symmetrical tensile vibration. In the PAm spectrum, due to the tensile vibration of $\mathrm{N}-\mathrm{H}$, the PAm curve has a characteristic peak at $3187 \mathrm{~cm}^{-1}$. Due to CONH2 stretching and $\mathrm{C}-\mathrm{N}$ stretching, PAm also showed characteristic peaks at 1647 and $1416 \mathrm{~cm}^{-1} .1046 \mathrm{~cm}^{-1}$ is the $\mathrm{C}=\mathrm{N}$ tensile vibration caused by MBA when PAm polymerizes. 

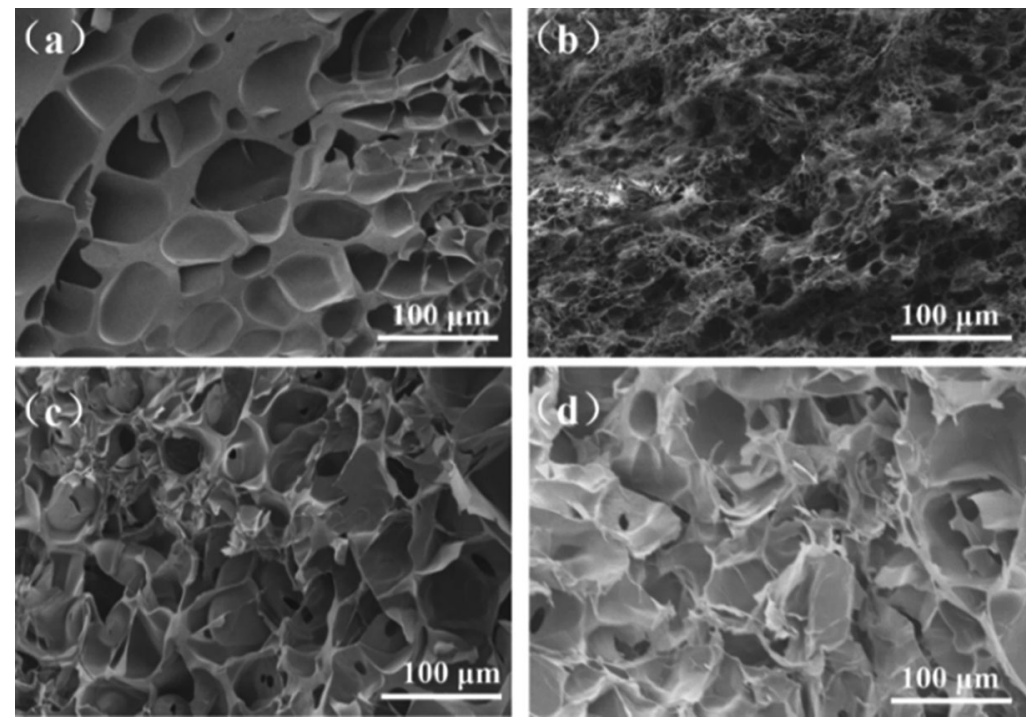

Figure 2: SEM images of hydrogels with different components: (a) PAm-SA; (b) PAm-PDA-SA; (c) PAmGO-SA; (d) PAm-prGO-SA hydrogel

(a)

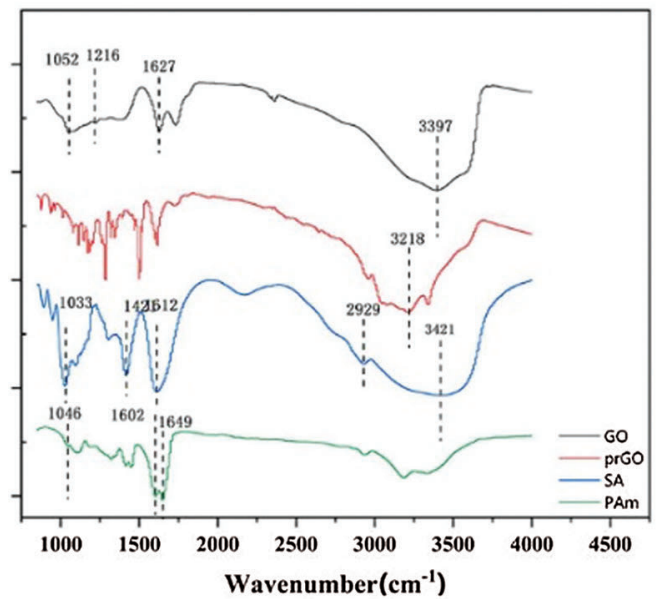

(c)

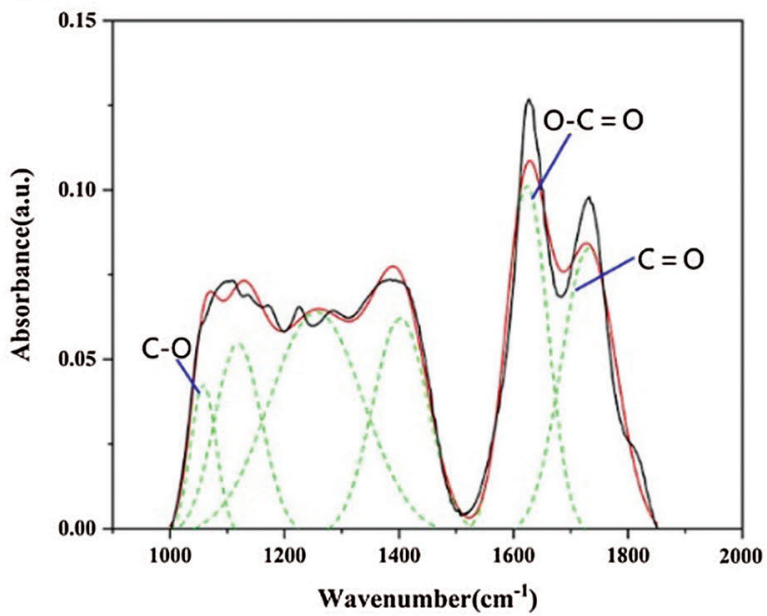

(b)

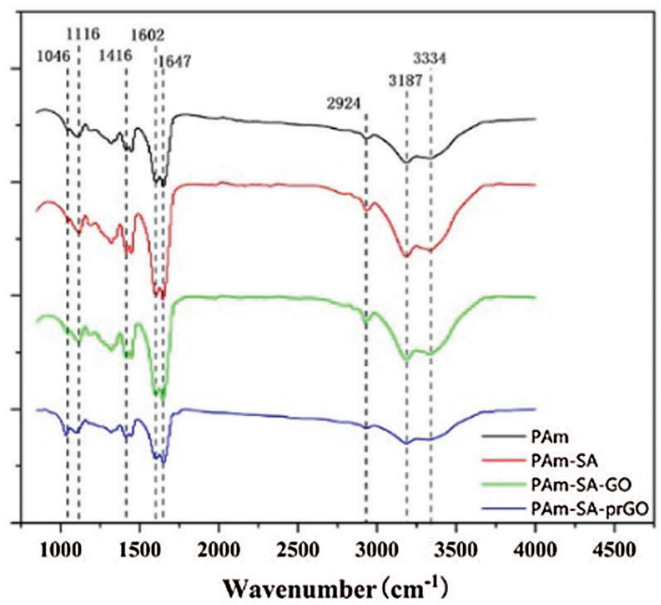

(d)

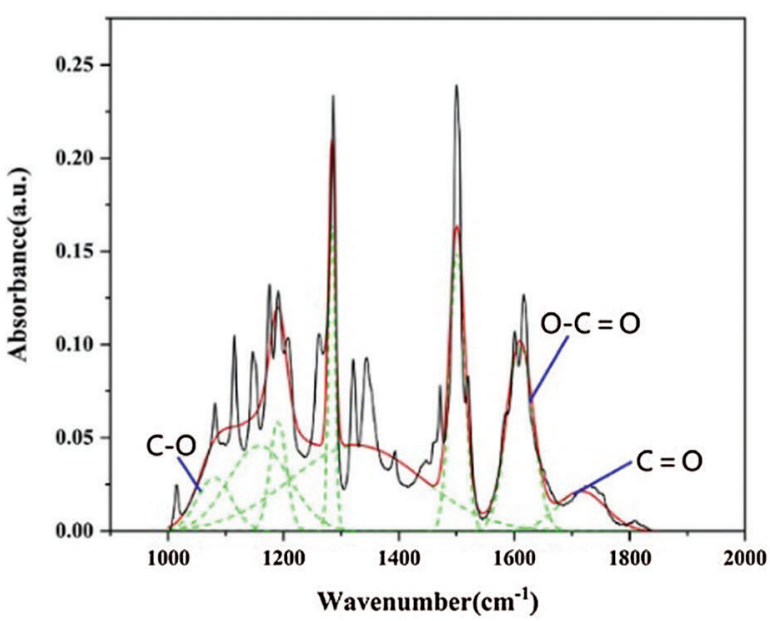

Figure 3: FTIR images of hydrogels: (a) different components (b) various hydrogels (c) GO; (d) prGO 
The FTIR results of SA/PAm and SA/PAm/GO are shown in Fig. $3 b, 2930 \mathrm{~cm}^{-1}$, and $2853 \mathrm{~cm}^{-1}$ correspond to the symmetric and antisymmetric stretching vibration absorption peaks of $\mathrm{CH}$, respectively. $1416 \mathrm{~cm}^{-1}$ and $1602 \mathrm{~cm}^{-1}$ are the symmetric and antisymmetric stretching vibration peaks of COO-. The peak at $3181 \mathrm{~cm}^{-1}$ is the symmetric and antisymmetric stretching vibration peaks of COO confirming the presence of the symmetrical vibration of $-\mathrm{NH}_{2}$. After SA and $\mathrm{GO}$ are added to PAm, the adsorption peak of $-\mathrm{OH}$ becomes broader and stronger, indicating that hydrogen bond could be formed in the between molecules. With the increase of GO, the in-plane bending vibration peak of the corresponding $\mathrm{NH}$ at $1647 \mathrm{~cm}^{-1}$ shifts to the long wavenumber direction, and the intensity increases. Due to the gap between the $\mathrm{OH}$ of the GO nanosheet and the $\mathrm{NH}$ on the PAm molecular chain. The hydrogen bonding interaction of the alkoxy $\mathrm{CO}$ at $1116 \mathrm{~cm}^{-1}$ shifts to the low wavenumber direction, and the intensity increases, indicated that there is a hydrogen bonding interaction between the dispersed GO and SA molecular chains, showed that in the process of In-situ polymerization, GO can participate in the formation of SA/PAm hydrogel network and change the gel network structure.

To determining the reduction of graphene oxide, Fourier transforms infrared (FTIR) was used to analyze the original GO and prGO after dopamine reduction. Figs. $3 \mathrm{c}$ and $3 \mathrm{~d}$ showed the FTIR spectra of GO and prGO. The spectra of GO nanosheets consist of multiple peaks in the range of $900-1800 \mathrm{~cm}^{-1}$, which could be assigned to various functional groups. For example, the absorption peaks at $1060 \mathrm{~cm}^{-1}$, $1113 \mathrm{~cm}^{-1}$, and $1365 \mathrm{~cm}^{-1}$ are vibration absorption peaks of $\mathrm{C}-\mathrm{O}-\mathrm{C}$ and $\mathrm{O}-\mathrm{C}=\mathrm{O}$, respectively. $\mathrm{C}=\mathrm{O}$ stretching vibration characteristic peak at $1741 \mathrm{~cm}^{-1}$ of GO carboxyl group [26-29]. All these proved that there were a lot of oxygen-containing groups connected to the GO hexagonal network. Therefore, we could determine the reduction degree of GO in the system by analyzing the change of the content of oxygen-containing functional groups in GO. From the FTIR spectra of prGO, it can be seen that the intensities of several peaks in the range of 900 to $1800 \mathrm{~cm}^{-1}$ are reduced. It indicated that the oxygencontaining groups on the GO surface are consumed during the reduction process. Tab. 2 showed that the reduction rate of oxygen-containing functional groups of graphene oxide is $51 \%$, indicated that PDA has successfully partially reduced GO. The presence of prGO is conducive to improving the conductivity of hydrogels.

Table 2: Peaks fitting results for Fourier transform infrared spectrum of pure GO and PDA-prGO

\begin{tabular}{lll}
\hline Functional groups & GO (\%) & prGO (\%) \\
\hline $\mathrm{C}=\mathrm{O}$ & 27.590 & 5.787 \\
$\mathrm{CO}$ & 5.072 & 5.541 \\
$\mathrm{O}-\mathrm{C}=\mathrm{O}$ & 20.479 & 14.652 \\
Reduction degree & & 51.128 \\
\hline
\end{tabular}

\subsection{Swelling and Water Retention Testing}

Fig. 4a showed the swelling properties of hydrogels of different components, indicated that the hydrogels with different ingredients showed excellent swelling properties. This also formed a mutual reflection with our discussion of the microstructure of hydrogels, from the SEM analysis, the reason why PAm-prGO-SA has a good swelling rate is that the hydrogel not only has a relatively uniform porosity, but also has a large pore diameter. PAm has excellent swelling properties due to its outstanding hydrophilicity and three-dimensional porous structure. The addition of SA and GO reduced the internal pores of the hydrogel. Therefore, the swelling properties of PAm-SA and PAm-GO-SA slightly decreased. Because DA can adhere to almost any molecular surface under alkaline conditions, and make it have 
adhesive and super hydrophilic properties. Moreover, the addition of PDA made the pores of the hydrogel denser and increased the porosity. Therefore, a small amount of unpolymerized DA in PAm-prGO-SA improves the swelling performance of the hydrogel compared with PAm-GO-SA [30].

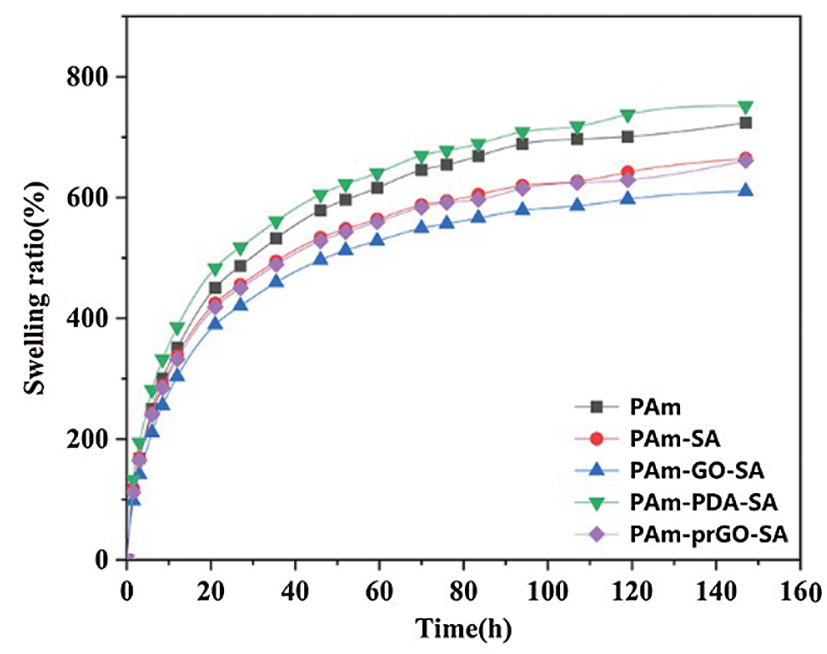

(a)

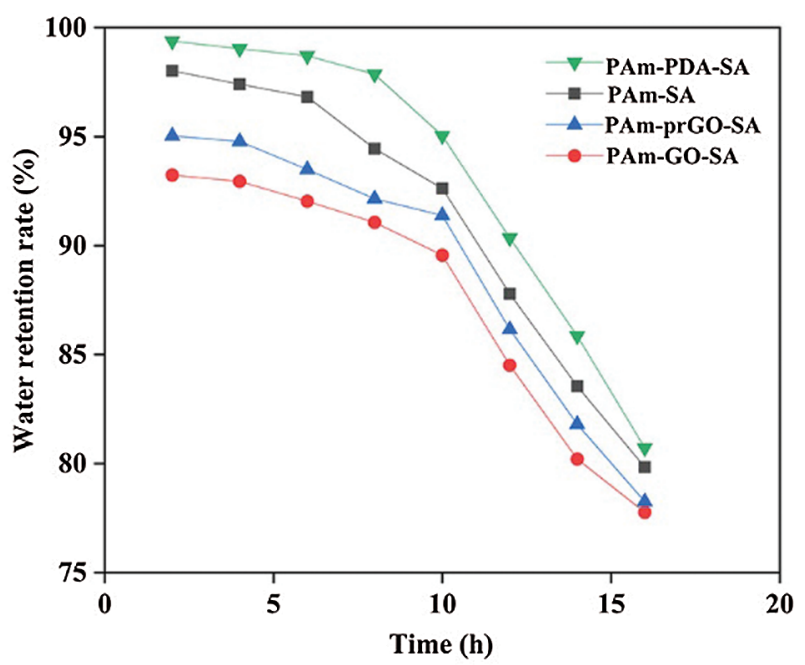

(b)

Figure 4: (a) Swelling ratio and (b) Water retention rate of hydrogels with different components

The dynamic water retention of different components of hydrogels was showed in Fig. 4b. According to the diagram, the water content of the hydrogel in the first $10 \mathrm{~h}$ only decreased slowly, and its value was above $90 \%$. However, the moisture in the hydrogel will dissipate with time and the moisture retention will gradually become worse. After $15 \mathrm{~h}$, the water content remained at about $80 \%$, indicated that the hydrogel had good water retention properties. PAm-prGO-SA hydrogel has good water retention, probably due to the spatial network structure of hydrogels and the large number of hydrophilic groups and synergistic effects on SA, GO and PDA chains.

\subsection{Mechanical Testing}

SA content is the key to the mechanical properties of PAm-SA interpenetrating polymer network hydrogel. SA has the characteristics of non-Newtonian fluid. Experiments showed that when the content of SA aqueous solution exceeds $4 \mathrm{wt} . \%$, its viscosity is too large, which is not conducive to the construction of a uniform hydrogel network. Fig. 5a is PAm-SA hydrogel prepared with SA content of 0 , 1,2 , and $3 \mathrm{wt} . \%$, respectively. PAm-SA hydrogels can improve the toughness of hydrogels due to the entanglement of SA chains in PAm single network. However, with the increase of SA content, the PAmSA hydrogel network and mechanical properties will also be affected.

Fig. 5e showed the stress-strain curves of PAm-SA hydrogels with different contents of SA. The tensile strength and elongation increased with the increase of SA content. Because the content of SA increased, the internal crosslinking network structure of hydrogel becomes more compact. When the hydrogel is stretched by an external force, the interwoven molecular chains inside can dissipate the action of the external force, which makes the hydrogel not easy to break. However, when the content of SA increased from $2 \mathrm{wt} . \%$ to 3 wt.\%, the elongation of PAm-SA hydrogel decreased. The reason is that the content of SA is too high, the crosslinking network of the hydrogel is too dense, and the molecular chains could not move in the hydrogel. Therefore, the hydrogel could not disperse the external force through the molecular chain, so it becomes brittle. In the follow-up experiments, we choose the content of SA as $2 \mathrm{wt} . \%$. 


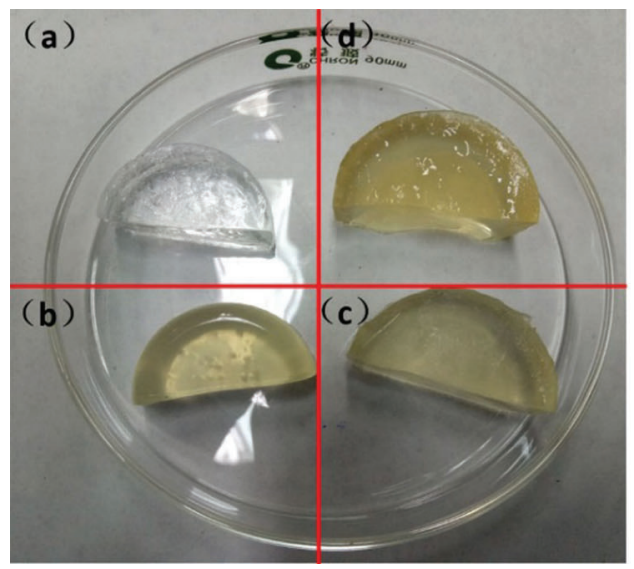

(e)

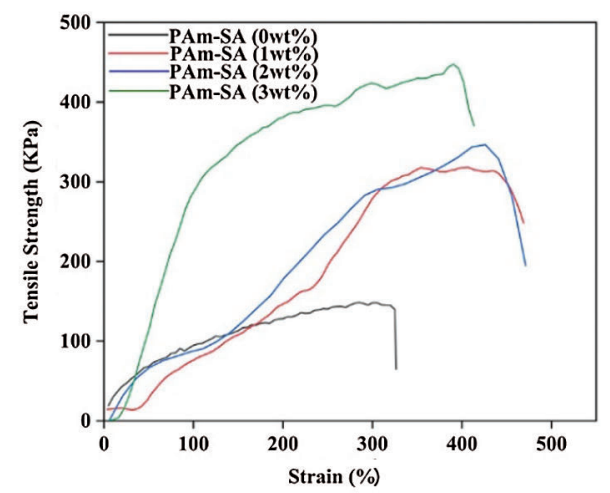

Figure 5: PAm-SA hydrogels prepared with different SA contents: (a) 0 wt.\%, (b) 1 wt.\%, (c) 2 wt.\%, (d) 3 wt.\%. (e) Stress-strain curves of PAm-SA hydrogels prepared with different SA contents

PAm-prGO-SA hydrogels prepared by adding PDA and GO into PAm-SA. The overall mechanical properties of hydrogels were changed with the addition of PDA and GO. Therefore, Figs. 6a and 6b showed the stress-strain curves of different components of hydrogels at room temperature by a universal testing machine. The tensile strength of PAm-SA, PAm-GO-SA, PAm-PDA-SA, and PAm-prGO-SA hydrogels were $270,852,636$, and $750 \mathrm{KPa}$, respectively. The fracture elongation was $615 \%, 780 \%$, $883 \%$ and $900 \%$, respectively. The tensile strength and elongation of PAm-SA hydrogels are lower than those of PAm-prGO-SA hydrogels due to the formation of strong hydrogen bonding, Van der Waals force, and pion stacking between the unreduced GO and PAm, PDA, and SA in the PAm-prGO-SA hydrogel, thus enhancing the toughness of the hydrogel. Because of the large amount of GO in the PAmGO-SA hydrogel, the network space of the hydrogel decreases, which makes it crisp, and the elongation decreases. However, due to the interaction force between GO and macromolecular chains, PAm-GO-SA hydrogel also obtained a higher tensile strength [31]. In PAm-PDA-SA hydrogel, PAm will entangle with PDA and SA molecular chains, and the external force will be dissipated evenly through the molecular chain, so the hydrogel has larger elongation. Fig. 6c showed Young's modulus of PAm-GO-SA hydrogel with different CNT concentrations, proved that CNT and PDA increase the toughness of hydrogels. In addition, the toughness of hydrogels often contributes greatly to energy dissipation, which is essential for the practical application of wound dressings [32]. For this purpose, cyclic loading and unloading tests of PAm-GO-SA hydrogels under $400 \%$ strain was carried out to evaluate their energy dissipation. As shown in Fig. 6d, obvious hysteresis loops were observed in the first experimental cycle, indicating that hydrogels could effectively dissipate energy. Under the cross-link effect of reversible covalent bond and hydrogen bond, PAm-GO-SA can produce energy dissipation through chain dissociation during the deformation of hydrogel, leading to a larger hysteresis loop. It was caused by the breakage and recovery of hydrogen bonds and dynamic Schiff bonds between SA and PAM chains when hydrogels are stretched and resumed. After the first test cycle, the following cycle was carried out for the same hydrogel sample. However, the subsequent cycles showed small hysteresis loops, reflecting limited energy dissipation. Tab. 3 showed the fracture energy of hydrogel with different compositions. The fracture energy of PAmprGO-SA hydrogel is about three times that of PAm-SA hydrogel, indicating that the hydrogel has good toughness. 


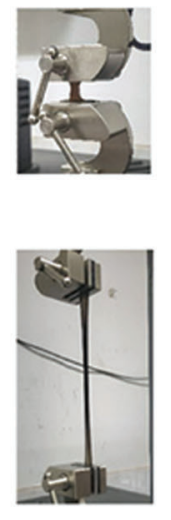

(a)

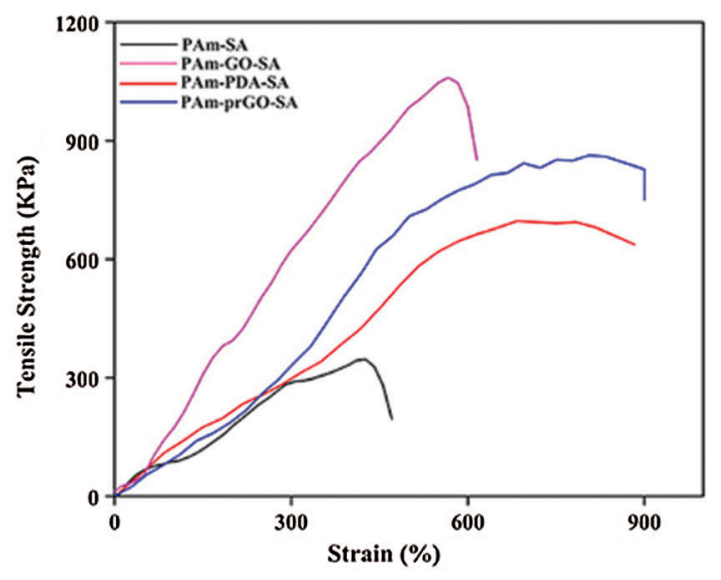

(b)

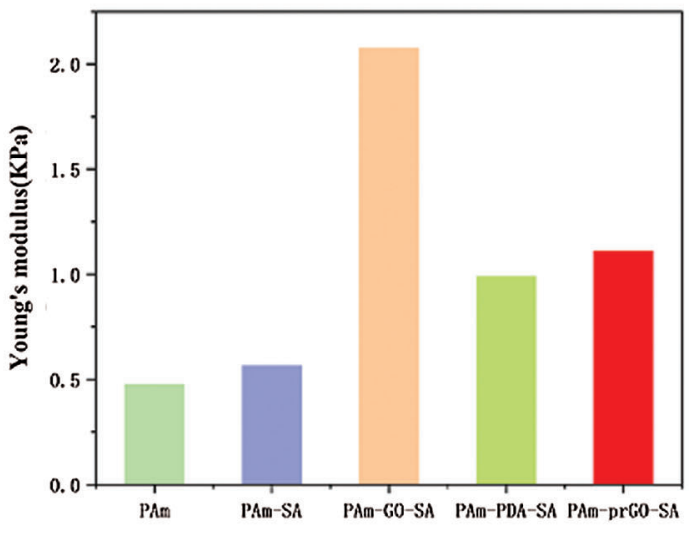

(c)

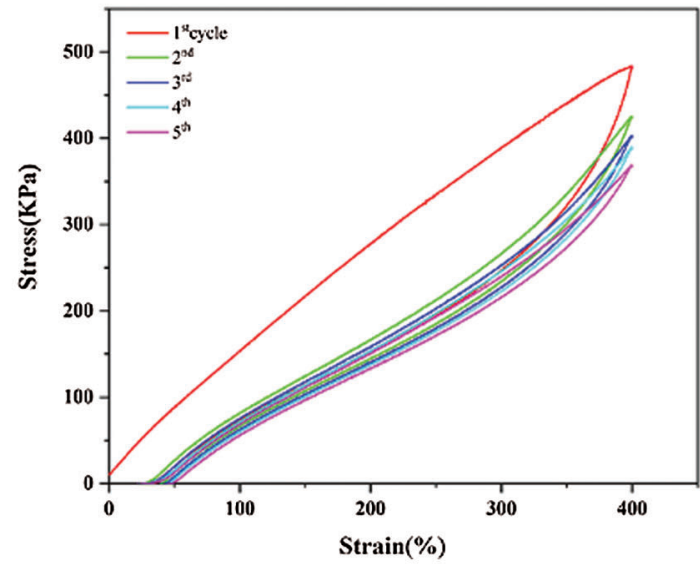

(d)

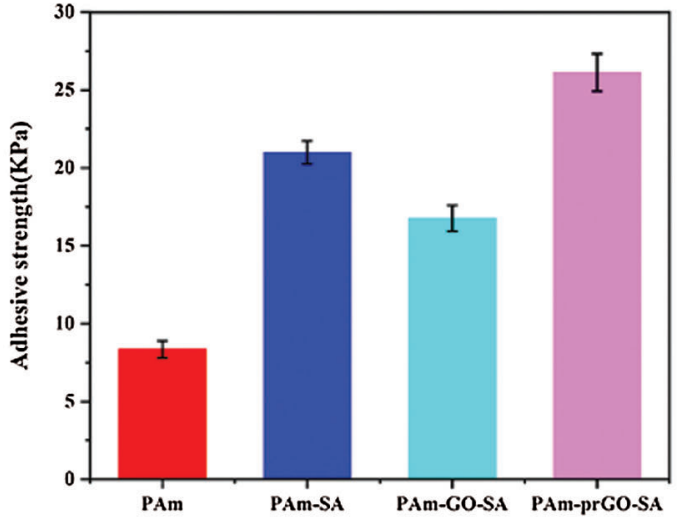

(e)

Figure 6: Tensile stress-strain curves measured by mechanical tensile test to test hydrogel of different components (a) and (b). (c) Young's modulus test of different compositions of hydrogels. (d) Loadingunloading tensile test of the PAm-PDA-SA hydrogel under $400 \%$ strain. (e) The adhesive strength of hydrogel with different components

Table 3: Fracture energies of hydrogels with different components

\begin{tabular}{lllll}
\hline Hydrogel & PAm-SA & PAm-GO-SA & PAm-PDA-SA & PAm-prGO-SA \\
\hline Fracture energy $\left(\mathrm{KJ} / \mathrm{m}^{3}\right)$ & 1527.93 & 3663.83 & 3854.39 & 4754.98 \\
\hline
\end{tabular}


PAm-prGO-SA hydrogel displayed high tissue adhesiveness and could easily adhere to skin tissue. The adhesive strength of different components of the hydrogel was tested between two pigskins. As shown in Fig. 6e, PAm hydrogel showed a weak adhesion. After the addition of SA and PDA, the adhesion of PAm-prGO-SA hydrogel increased, and the adhesion to pigskin reached $26.12 \pm 1.21 \mathrm{kPa}$. The adhesion of PAm-prGO-SA hydrogel was mainly attributed to the following two aspects. Firstly, SA has excellent adhesion. Secondly, because catechol groups on PDA exhibit a high affinity for various nucleophilic agents (such as amines, thiols, and imidazolines), PAm-prGO-SA hydrogels can bind to peptides and proteins on the surface of everything [33]. Therefore, PAm-prGO-SA has excellent adhesion under the interaction of viscosity, hydrogen bond, and $\pi-\pi$ bond of PDA and SA.

\subsection{Electro-Conductivity and Sensing Performance Analysis of PAm-prGO-SA Hydrogel}

The PAm-prGO-SA hydrogel not only possessed excellent mechanical properties but also presented outstanding electrical response capability due to the existence of prGO in the hydrogels. Because prGO came from the reduction of GO by PDA. Therefore, we can control the content of prGO in the PAm-prGO-SA hydrogel system by dominating the content of GO. According to Fig. 8a, the conductivity of the hydrogel increased with the rise in GO content. When the GO content was $0.25 \mathrm{wt} \%$, the conductivity of the hydrogel was as high as $12.99 \mathrm{~S} \mathrm{~cm}^{-1}$, showing excellent electrical properties. Figs. $7 \mathrm{~b}$ and $7 \mathrm{c}$ showed the bending sensing response and gauge factor of PAm-prGO-SA hydrogel with different GO content. The calculation method of gauge factor is provided by the existing literature [33]. With the increase of GO, the sensing response first increases and then decreases. When GO content is $0.2 \mathrm{wt} . \%$, the bending sensing response was $290 \%$ and the gauge factor was 2.5 , which indicated that the sensor has an outstanding response to the external force. However, with the increase of GO to $0.25 \mathrm{wt} . \%$, the density of prGO in hydrogels is too large. It may accumulate in cross-linked networks, resulting in uneven PAm-prGO-SA formation, which affects the sensor's response performance. Therefore, $0.2 \mathrm{wt} \%$ was selected as the optimal content of go in PAm prGO-SA. To verify the reliability of PAm-prGO-SA hydrogel, we carried out the loading/unloading experiment of hydrogel under the strain of $300 \%$ by using the mechanical testing machine in $1 \mathrm{~h}$ (Fig. 7d). Fig. 7e shows the current changes of the sensor under ten repeated loading/unloading cycles. It can be clearly observed that every loading/unloading cycle presents an almost repeated waveform, which indicates that the sensor has good cycle stability and reliability.

PAm-prGO-SA flexible hydrogels with copper sheets at both ends were connected to electrochemical workstations and computers to monitor resistance changes caused by various human movements. Fig. 8 shows the sensor response curve assembled into a wearable sensor for detecting joint activity and breathing of the human body. Figs. $8 \mathrm{a}-8 \mathrm{c}$ are the sensor response curves measured by applying the hydrogel to the elbow joint, knee joint, and neck of the human body through designated actions. Under different bending angles, the resistance of the hydrogel changes, while it was conversely increased to the original value when restored. By repeatedly performing the specified actions, it can be observed that each round has a similar waveform, and the current signal can be completely restored to the original value after each bending cycle, showing the excellent cycle stability and reliability of the sensor. The above experiment shows that the sensor can successfully monitor joint activity. It is expected to be used in continuous monitoring of medical rehabilitation training of fracture patients. In addition to large-scale motion monitoring, the sensor can detect small human motions due to its high electrical sensitivity. Fig. 8d shows the sensor placed on the subject's chest to measure changes in the thorax due to breathing. The sensor is subjected to a small external force, which changes the internal resistance of the hydrogel, thus forming a regular respiratory sensing response signal. For these regular movements, the conductivity of the sensor shows almost uniform peaks and shapes. It indicates that the electrical properties of the 

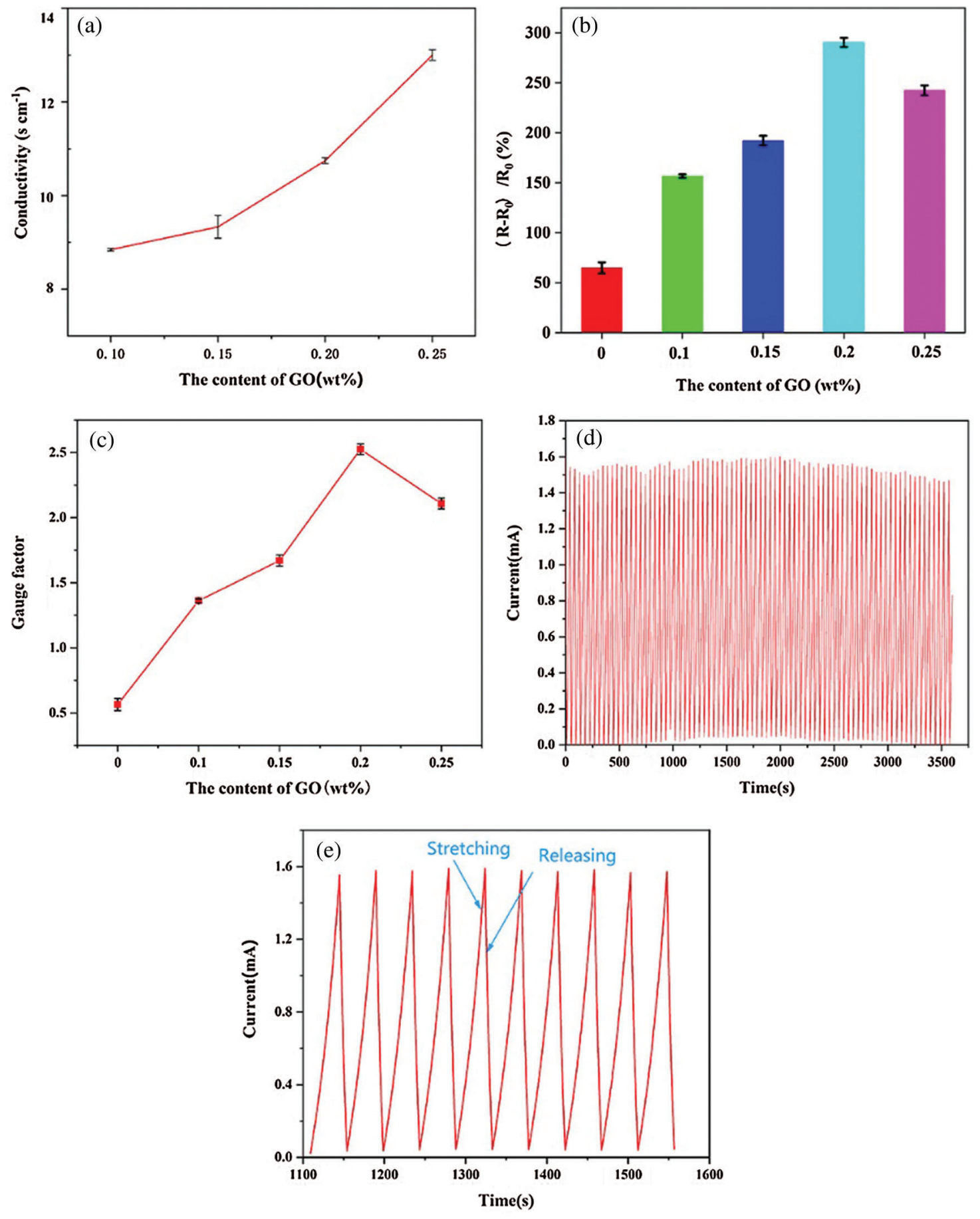

Figure 7: (a) Conductivity of hydrogels with different GO content. (b) Sensing response of PAm-prGO-SA hydrogel with different GO content. (c) Gauge factor of PAm-prGO-SA hydrogel with different GO content. (d) Real-time I-t curves measured by cyclic stretching/releasing at $5 \mathrm{~V}$ within $1 \mathrm{~h}$ for repeatability. (e) Zoomed-in version of the plot Fig. 7c from 1100-1550 s tests 
prepared sensor have good repeatability and stability. This combination of high sensitivity, electrical stability, and mechanical flexibility gives hydrogel strain sensors a promising application in wearable electronic products.
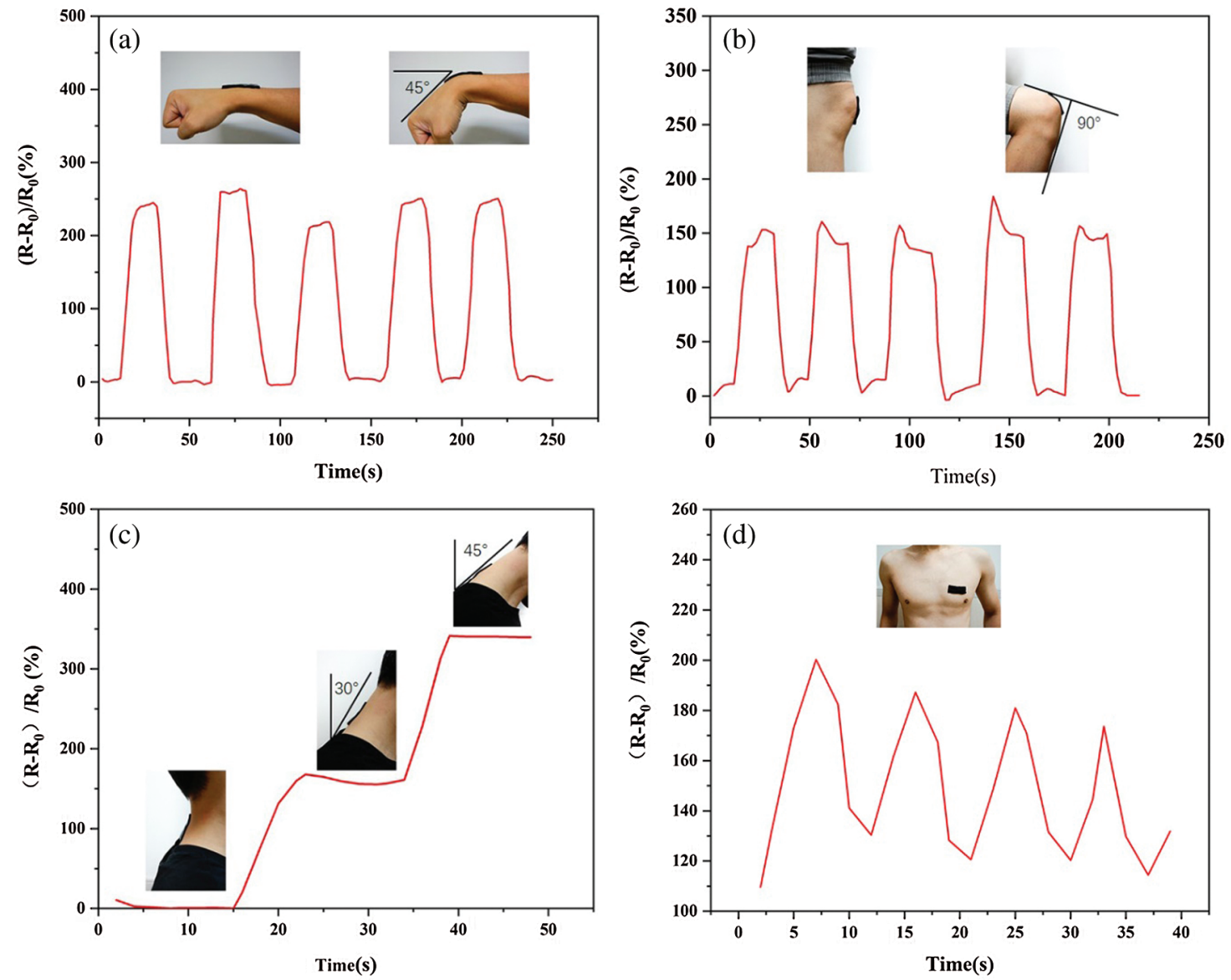

Figure 8: Flexible sensor assembled by PAm-prGO-SA hydrogel was used to measure human motion: (a) wrist joint; (b) knee joint; (c) neck joint; (d) thoracic activity caused by breathing

\subsection{Cytocompatibility}

Viability, proliferation, and cytotoxicity of NIH3T3 cells in PAm-prGO-SA hydrogel soaking solutions were evaluated using the CCK-8 method. It mainly includes observation of NIH3T3 cell growth morphology and CCK-8 cytotoxicity test. NIH3T3 cells were cultured in an incubator for 24,36 , and $48 \mathrm{~h}$. Figs. $9 \mathrm{a}$ and $9 \mathrm{~b}$ showed the growth morphology of NIH3T3 cells (test group and control group) after $36 \mathrm{~h}$ of culture. The results showed that NIH3T3 cells are spindle-shaped, which is the growth morphology of NIH3T3 cells under normal conditions [34]. It indicates that PAm-prGO-SA hydrogel has no side effects on the growth of NIH3T3 cells. Fig. 9c was the CCK-8 experiment conducted after cell culture for 24, 36, and $48 \mathrm{~h}$. Data analysis showed that the cytotoxicity of PAm-prGO-SA hydrogel is 0 grade because of RGR $>100$ in the test group. It confirmed that PAm-prGO-SA hydrogel is not toxic to NIH3T3 cells and has good biocompatibility. 


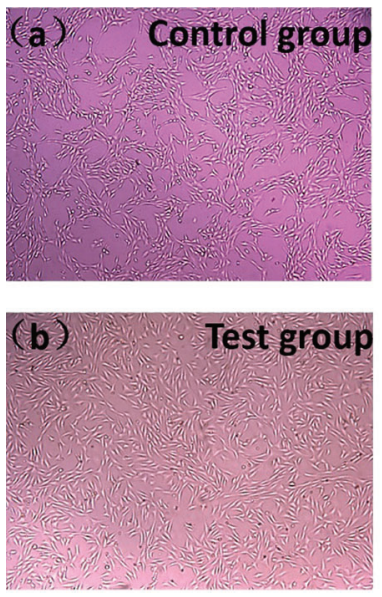

(c)

Figure 9: CCK-8 test results of PAm-prGO-SA hydrogel. (a) control group, (b) test group cell morphology of the experimental group after $36 \mathrm{~h}$ of culture; (c) relative growth rates of NIH3T3 fibroblasts cultured for 24, 36 and $48 \mathrm{~h}$, respectively

\section{Conclusions}

In summary, a novel type of high toughness and electro-conductive PAm-prGO-SA composite hydrogel with excellent water retention was successfully prepared via a facile combined one-pot preparation process. The whole synthesis process is convenient with high repeatability. Firstly, PAm was synthesized by selfpolymerization of AM and formed a semi-interpenetrating network with the SA chain. Secondly, a large number of hydrogen bonds and $\pi-\pi$ bonds are formed among PAm, SA, GO, and PDA. The dual functions of physical winding and chemical bonds give the PAm-prGO-SA hydrogel super high moisture retention, breaking tensile rate $(900 \%)$, tensile strength $(750 \mathrm{KPa})$, and adhesion strength $(26.12 \mathrm{kPa})$. The presence of prGO has become the essential factor supporting the hydrogel with excellent conductivity (12.99 $\mathrm{S} \mathrm{cm}^{-1}$ ) and sense response (290\%). These advantages and excellent biocompatibility make PAm-prGO-SA hydrogels an ideal material for making flexible wearable sensors. The PAm-prGOSA hydrogel-based sensors could not only quickly, repeatedly, and accurately respond to large-scale movements of human joints such as elbows, neck, and knees, but also respond to micro-movements such as breathing. The PAm-prGO-SA hydrogel-based sensors can be used as a wearable detection electrode for medical monitoring devices to achieve real-time and efficient detection of physiological signals of patients. Therefore, in the future, The PAm-prGO-SA hydrogel-based sensors are expected to be applied to flexible wearable medical monitoring devices.

Funding Statement: This research is supported by Chongqing postgraduate research and innovation project in 2019 (No. CYS19217).

Conflicts of Interest: The authors declare that they have no conflicts of interest to report regarding the present study.

\section{References}

1. Zheng, C., Lu, K., Lu, Y., Zhu, S., Yue, Y. et al. (2020). A stretchable, self-healing conductive hydrogels based on nanocellulose supported graphene towards wearable monitoring of human motion. Carbohydrate Polymers, 250, 116905. DOI 10.1016/j.carbpol.2020.116905. 
2. Huang, S. Q., Su, S. Y., Gan, H. B., Wu, L. J., Lin, C. H. et al. (2019). Facile fabrication and characterization of highly stretchable lignin-based hydroxyethyl cellulose self-healing hydrogel. Carbohydrate Polymers, 223, 115080. DOI 10.1016/j.carbpol.2019.115080.

3. Chen, J. P., Chen, S. H., Lai, G. J. (2012). Preparation and characterization of biomimetic silk fibroin/chitosan composite nanofibers by electrospinning for osteoblasts culture. Nanoscale Research Letters, 7, 170. DOI 10.1021/acsnano.8b07159.

4. Ramachandran, V., Bartlett, M. D., Wissman, J., Majidi, C. (2016). Elastic instabilities of a ferroelastomer beam for soft reconfigurable electronics. Extreme Mechanics Letters, 9, 282-290. DOI 10.1016/j.eml.2016.08.007.

5. Lin, N., Dufresne, A. (2014). Nanocellulose in biomedicine: Current status and future prospect. European Polymer Journal, 59, 302-325. DOI 10.1016/j.eurpolymj.2014.07.025.

6. Li, Y., Zheng, L., Wang, X. (2019). Flexible and wearable healthcare sensors for visual reality health-monitoring. Virtual Reality \& Intelligent Hardware, 1(4), 411-427. DOI 10.1016/j.vrih.2019.08.001.

7. Wei, X. P., Luo, Y. L., Xu, F., Chen, Y. S. (2016). Sensitive conductive polymer composites based on polylactic acid filled with multiwalled carbon nanotubes for chemical vapor sensing. Synthetic Metals, 215, 216-222. DOI 10.1016/j.synthmet.2016.02.023.

8. Niu, J., Wang, J., Dai, X., Shao, Z., Huang, X. (2018). Dual physically crosslinked healable polyacrylamide/ cellulose nanofibers nanocomposite hydrogels with excellent mechanical properties. Carbohydrate Polymers, 193, 73-81. DOI 10.1016/j.carbpol.2018.03.086.

9. Yang, J., Zhao, J. J., Xu, F., Sun, R. C. (2013). Revealing strong nanocomposite hydrogels reinforced by cellulose nanocrystals: Insight into morphologies and interactions. ACS Applied Materials \& Interfaces, 5, 12960-12967. DOI 10.1021/am403669n.

10. Dai, X., Zhang, Y., Gao, L., Bai, T., Wang, W. et al. (2015). A mechanically strong, highly stable, thermoplastic, and self-healable supramolecular polymer hydrogel. Advanced Materials, 27, 3566-3571. DOI 10.1002/ adma.201500534.

11. Xie, C., Wang, X., He, H., Ding, Y., Lu, X. (2020). Mussel-inspired hydrogels for self-adhesive bioelectronics. Advanced Functional Materials, 30, 1909945. DOI 10.1002/adfm.201909954.

12. Cataldi, P., Dussoni, S., Ceseracciu, L., Maggiali, M., Bayer, I. S. et al. (2018). Carbon nanofiber vs. graphenebased stretchable capacitive touch sensors for artificial electronic skin. Advanced Science, 5(2), 1870011. DOI 10.1002/advs.201700587.

13. Muthoosamy, K., Manickam, S. (2017). State of the art and recent advances in the ultrasound-assisted synthesis, exfoliation and functionalization of graphene derivatives. Ultrasonics Sonochemistry, 39, 478-493. DOI 10.1016/ j.ultsonch.2017.05.019.

14. Agnelli, S., Baldi, F., Bignotti, F., Salvadori, A., Peroni, I. (2018). Fracture characterization of hyperelastic polyacrylamide hydrogels. Engineering Fracture Mechanics, 203, 54-65. DOI 10.1016/j. engfracmech.2018.06.004.

15. Sun, J. Y., Zhao, X., Illeperuma, W. R., Chaudhuri, O., Oh, K. H. et al. (2012). Highly stretchable and tough hydrogels. Nature, 489, 133-136. DOI 10.1038/nature11409.

16. Zheng, Q., Zhao, L., Wang, J., Wang, S., Liu, Y. et al. (2020). High-strength and high-toughness sodium alginate/ polyacrylamide double physically crosslinked network hydrogel with superior self-healing and self-recovery properties prepared by a one-pot method. Colloids and Surfaces A: Physicochemical and Engineering Aspects, 589, 124402. DOI 10.1016/j.colsurfa.2019.124402.

17. Bai, Y. Y., Lei, Y. H., Shen, X. J., Luo, J., Yao, C. L. et al. (2017). A facile sodium alginate-based approach to improve the mechanical properties of recycled fibers. Carbohydrate Polymers, 174, 610-616. DOI 10.1016/j. carbpol.2017.06.091.

18. Yang, X., Niu, X., Mo, Z., Guo, R., Liu, N. et al. (2019). Electrochemical chiral interface based on the michael addition/Schiff base reaction of polydopamine functionalized reduced graphene oxide. Electrochimica Acta, 319, 705-715. DOI 10.1016/j.electacta.2019.07.040. 
19. Di, X., Kang, Y., Li, F., Yao, R., Chen, Q. et al. (2019). Poly(n-isopropylacrylamide)/polydopamine/clay nanocomposite hydrogels with stretchability, conductivity, and light/thermo dual responsive bending and adhesive properties. Colloids \& Surfaces B Biointerfaces, 177, 149-159. DOI 10.1016/j.colsurfb.2019.01.058.

20. Yue, Y., Wang, X., Han, J., Yu, L., Chen, J. et al. (2018). Effects of nanocellulose on sodium alginate/ polyacrylamide hydrogel: Mechanical properties and adsorption-desorption capacities. Carbohydrate Polymers, 206, 289-301. DOI 10.1016/j.carbpol.2018.10.105.

21. Pan, X., Zuo, G., Su, T., Cheng, S., Gu, Y. et al. (2018). Polycarboxylic magnetic polydopamine sub-microspheres for effective adsorption of malachite green. Colloids \& Surfaces A: Physicochemical \& Engineering Aspects, 560, 106-113. DOI 10.1016/j.colsurfa.2018.10.014.

22. He, Y., Cui, H. (2012). Synthesis of highly chemiluminescent graphene oxide/silver nanoparticle nano-composites and their analytical applications. Journal of Materials Chemistry, 22(18), 9086-9091. DOI 10.1039/C2JM16028E.

23. Bahrami, Z., Akbari, A., Eftekhari-Sis, B. (2019). Double network hydrogel of sodium alginate/polyacrylamide cross-linked with poss: Swelling, dye removal and mechanical properties. International Journal of Biological Macromolecules, 129, 187-197. DOI 10.1016/j.ijbiomac.2019.02.046.

24. Liu, S., Bastola, A. K., Li, L. (2017). A 3D printable and mechanically robust hydrogel based on alginate and graphene oxide. ACS Applied Materials \& Interfaces, 9, 41473-41481. DOI 10.1021/acsami.7b13534.

25. Sharma, N., Sharma, V., Vyas, R., Kumari, M., Kaushal, A. et al. (2019). A new sustainable green protocol for production of reduced graphene oxide and its gas sensing properties-sciencedirect. Journal of Science: Advanced Materials and Devices, 4(3), 473-482. DOI 10.1016/j.jsamd.2019.07.005.

26. Drewniak, S., Muzyka, R., Stolarczyk, A., Pustelny, T., Kotyczka-Moranska, M. et al. (2016). Studies of reduced graphene oxide and graphite oxide in the aspect of their possible application in gas sensors. Sensors, 16(1), 103. DOI 10.3390/s16010103.

27. Arthi, G. P. B., Bd, L. (2015). A simple approach to stepwise synthesis of graphene oxide nanomaterial. Journal of Nanomedicine \& Nanotechnology, 6(1), 1000253. DOI 10.4172/2157-7439.1000253.

28. Liu, R., Zhang, H., Zhang, F., Wang, X., Zhang, Y. (2018). Polydopamine doped reduced graphene oxide/ mesoporous silica nanosheets for chemo-photothermal and enhanced photothermal therapy. Materials Science and Engineering C, 96, 138-145. DOI 10.1016/j.msec.2018.10.093.

29. Esmat, M., Farghali, A. A., Khedr, M. H., El-Sherbiny, I. M. (2017). Alginate-based nanocomposites for efficient removal of heavy metal ions. International Journal of Biological Macromolecules, 102, 272-283. DOI 10.1016/j. ijbiomac.2017.04.021.

30. Suneetha, M., Rao, K. M., Han, S. S. (2019). Mussel-inspired cell/tissue-adhesive, hemostatic hydrogels for tissue engineering applications. ACS Omega, 4, 12647-12656. DOI 10.1021/acsomega.9b01302.

31. Yang, Y., Song, S., Zhao, Z. (2017). Graphene oxide (GO)/polyacrylamide (PAM) composite hydrogels as efficient cationic dye adsorbents. Colloids and Surfaces A: Physicochemical and Engineering Aspects, 513, 315-324. DOI 10.1016/j.colsurfa.2016.10.060.

32. Gong, Z., Zhang, G., Zeng, X., Li, J., Li, G. et al. (2016). High-strength, tough, fatigue resistant, and self-healing hydrogel based on dual physically cross-linked network. ACS Applied Materials \& Interfaces, 8, 24030-24037. DOI 10.1021/acsami.6b05627.

33. Wang, X., Liu, X., Schubert, D. W. (2021). Highly sensitive ultrathin flexible thermoplastic polyurethane/carbon black fibrous film strain sensor with adjustable scaffold networks. Nano-Micro Letters, 13(1), 1-19. DOI 10.1007/ s40820-021-00592-9.

34. Zhang, Y., Liang, B., Jiang, Q., Li, Y., Feng, Y. et al. (2020). Flexible and wearable sensor based on graphene nanocomposite hydrogels. Smart Materials and Structures, 29(7), 057027. DOI 10.1088/1361-665X/ab89ff. 